\section{(2) \\ BRAZIULIAN JOURNAL \\ OF MEDICAL AND BIOLOGICAL RESEARCH}

www.bjournal.com.br
ISSN 0100-879X

Volume 45 (5) 376-472 May 2012

BIOMEDICAL SCIENCES

AND

CLINICAL INVESTIGATION

Braz J Med Biol Res, May 2012, Volume 45(5) 386-391

doi: 10.1590/S0100-879X2012007500048

Heparan sulphate, its derivatives and analogues share structural characteristics that can be exploited, particularly in inhibiting microbial attachment

T.R. Rudd, A. Hughes, J. Holman, E. de Oliveira Ferreira, R.M. Cavalcante Pilotto Domingues and E.A. Yates

The Brazilian Journal of Medical and Biological Research is partially financed by

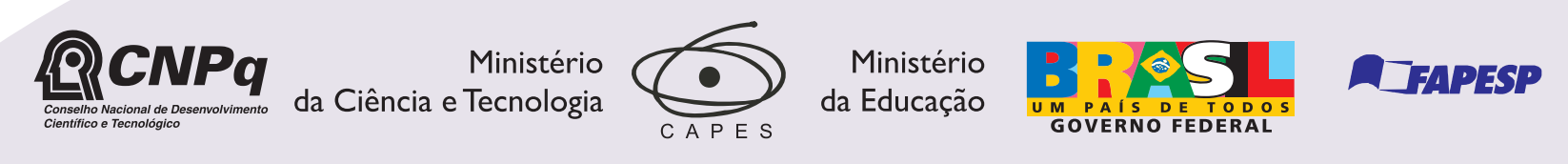

Scie/O
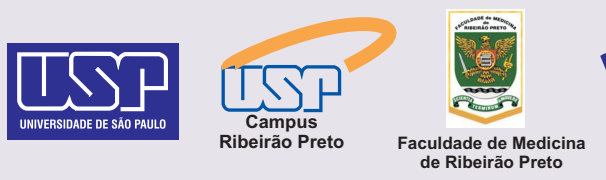

Institutional Sponsors

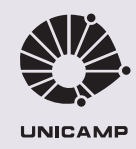

SHIMADZu

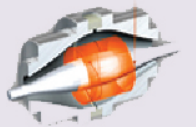

FAEPA

HCFMRP

UNICAMP
GOVERNO FEDERAL 


\title{
Heparan sulphate, its derivatives and analogues share structural characteristics that can be exploited, particularly in inhibiting microbial attachment
}

\author{
T.R. Rudd ${ }^{1}$, A. Hughes ${ }^{2}$, J. Holman², E. de Oliveira Ferreira ${ }^{3,4}$, \\ R.M. Cavalcante Pilotto Domingues ${ }^{3}$ and E.A. Yates $^{2}$ \\ ${ }^{1}$ Istituto di Chimica e Biochimica "G. Ronzoni", Milano, Italy \\ ${ }^{2}$ Chemical and Structural Biology, Institute of Integrative Biology, \\ University of Liverpool, Liverpool, UK \\ ${ }^{3}$ Laboratório de Biologia de Anaeróbios, Centro de Ciências da Saúde, \\ Universidade Federal do Rio de Janeiro, Rio de Janeiro, RJ, Brasil \\ ${ }^{4}$ Universidade Federal do Rio de Janeiro, Polo Xerém, Rio de Janeiro, RJ, Brasil
}

\begin{abstract}
Heparan sulphate (HS) and the related polysaccharide, heparin, exhibit conformational and charge arrangement properties, which provide a degree of redundancy allowing several seemingly distinct sequences to exhibit the same activity. This can also be mimicked by other sulphated polysaccharides, both in overall effect and in the details of interactions and structural consequences of interactions with proteins. Together, these provide a source of active compounds suitable for further development as potential drugs. These polysaccharides also possess considerable size, which bestows upon them an additional useful property: the capability of disrupting processes comprising many individual interactions, such as those characterising the attachment of microbial pathogens to host cells. The range of involvement of HS in microbial attachment is reviewed and examples, which include viral, bacterial and parasitic infections and which, in many cases, are now being investigated as potential targets for intervention, are identified.
\end{abstract}

Key words: Heparan sulfate; Biosynthesis; Structure-function; Microbial attachment

\section{Introduction}

Heparan sulphate (HS) proteoglycans are present on the cell surface and in the extracellular matrix (ECM) of practically all mammalian cells, although the sequence can vary considerably between tissues, individuals and species. HS regulates many important biochemical and developmental processes, including cell signalling (e.g., via the fgf-fgf receptor tyrosine kinase pathway) but is also implicated in a number of disease processes, from Alzheimer's to the attachment of microbial pathogens such as dengue virus, meningococcus and parasites. HS possesses a domain structure comprising sections of repeating 1,4 linked $\beta$-GlcA$\alpha-G \mid c N A c$, termed NA domains, alternating with more highly sulphated sections containing IdoA2S-GIcNS+/-6S, termed $S$ domains. The $\alpha-L-I d o A$ residues are generated by epimerisation at C-5 of $\beta-D-G \mid c A$ and are flanked in turn by stretches of GlcNS and GlcNAc+/-6S residues. HS, which is expressed on the cell surfaces of all mammalian cells and in the ECM, interacts with many proteins and is at the hub of extensive signalling networks in multicellular animals (1-3). These interactions in their entirety have been called the "HS-interactome" and are considered to regulate fundamental biochemical and developmental processes in multicellular organisms (2).

Heparin is a close structural analogue of HS, which

Correspondence: E.A. Yates, Chemical and Structural Biology, Institute of Integrative Biology, University of Liverpool, Crown Street, Liverpool L69 7ZB, Great Britain. E-mail: eayates@liv.ac.uk

Presented at the XL Annual Meeting of the Sociedade Brasileira de Bioquímica e Biologia Molecular, Foz do Iguaçu, PR, Brazil, April 30-May 3, 2011.

Received December 21, 2011. Accepted March 20, 2012. Available online April 5, 2012. Published May 7, 2012. 
is produced by mast cells and possesses a comparable underlying repeating structure comprising 1,4 linked uronate-glucosamine units but does not have the domain structure of HS, in which more sulphated and L-IdoA-rich stretches, termed S-domains, are interspersed with longer, less-sulphated regions called NA domains. There are also stretches with intermediate levels of sulphation in between these more highly sulphated regions. Heparin, in contrast, is more sulphated than $\mathrm{HS}$ and has a higher content of L-IdoA.

Despite extensive efforts over several decades, a clear understanding of $\mathrm{HS}$ biosynthesis, particularly its regulation, and the nature of the relationship between sequence and biological activity have remained elusive. Early work regarding the latter, presumably following the proven approach established for proteins, strove to uncover HS specificity based on the presumption of a direct correspondence between the unique primary sequence and biological activity. The apparent specificity of the antithrombin (AT) binding sequence in heparin, which arose from the first detailed work to reveal the apparent binding requirements for an HS structure for a particular protein - a pentasaccharide $(4,5)$ - reinforced this tendency to concentrate on the search for highly specific sequences (4-6).

\section{Exquisite specificity challenged}

Despite this apparent early breakthrough, in the intervening years, very few other examples of highly specific HSprotein interactions have come to light. Instead, a general picture of HS structure-activity relationships has emerged in which several sequences exhibit similar binding and/ or activity while the same sequence can support several activities. Sequences with comparable activities may not even share a common substitution pattern. Evidence for these assertions, of which there is now a considerable body, continues to confound efforts to uncover highly specific, one sequence-one activity relationships for HS activity.

An alternative explanation has been proposed to explain these characteristics. This is based on the idea that an HS sequence has certain physical chemical properties, which can be summarised as charge distribution, shape and flexibility, and these can be replicated either precisely, or (more likely) sufficiently closely, to bestow the same or very similar activity on the sequence. For example, it has been recently demonstrated that not only sugars based on the common backbone of HS and heparin but also other, seemingly unrelated sulphated polysaccharides, can mimic the necessary physical properties and, moreover, can induce the same structural changes and stabilisation in target proteins to replicate the biological activities of HS (7). This ability is not restricted solely to a few serendipitous cases but appears to be a general characteristic of this molecular system. Added to this is the observation that, among structurally distinct derivatives of HS or heparin derivatives (which serve as a proxy for HS), there is ample demonstration of redundancy between substitution pattern (or sequence) and biological activity, suggesting that a change in emphasis in the search for the nature of HS specificity is due.

The validity of the concepts of redundancy between HS sequences and activity, and the effects of cations on their binding properties is illustrated by the binding profiles of phage display antibodies to HS structures and HS analogues based on a heparin backbone and on cell surface HS (Solari V, Rudd TR, Guimond SE, Skidmore MA, Siligardi G, van der Westerloo EM, et al., unpublished results). The binding of phage display antibodies to heparin analogues of HS was shown to be sensitive to exposure to cations, phage display antibody binding both increasing and decreasing in a complex manner, depending on the polysaccharide in question, and on the cation type. Furthermore, the effect was also shown on cell surface HS. Phage display antibodies, which bind HS via a short recognition sequence comprising several amino acids, appear to be similar to proteins in this respect.

In light of these observations, the quest for the separation of individual oligosaccharides and subsequent measurement of their activities, undertaken in the hope of uncovering a simple correlation between sequence (or substitution pattern) and activity, seems destined to fail. These activities are often presented as searches among highly diverse libraries but are frequently actually a search for such correlations among a set of compounds of very restricted diversity, and this continues to confuse investigators. In only one instance of which we are aware has a search been made through a series of sequences approaching maximum sequence diversity (8).

With the above points in mind, the following provisos should be appreciated in the search for HS structure-activity relationships: 1) correlations between activity and substitution pattern cannot be understood using a purely reductionist approach, while ignoring conformational changes that may occur in the molecule resulting from these changes in substitution; 2) correlations between activity and sequence, from which over-arching conclusions are extrapolated, have little value if based on very restricted sequence diversity among the sequences being tested, unless it is established that only those sequences are present in vivo in connection with the activity in question. A corollary to this is the need to have a realistic understanding of the potential sequence diversity and that actually observed in a sample.

\section{Redundancy in heparan sulphate sequence-activity relationships}

The ability of several distinct sequences to impart similar, or identical, activity with a particular protein may have implications for our understanding of $\mathrm{HS}$ biosynthesis. Formerly, it was assumed that particular sequences would need to be synthesised and their synthesis controlled but 
this may not be necessary in the way previously understood; generation of one or more functional structures may be all that is required for a particular activity and, potentially, these could comprise a number of quite different sequences and substitution patterns.

One aspect that has not yet been explored in much depth is the possibility that the activity profile of HS sequences, rather than their individual roles, may be the defining activity characteristic. Thus, it will be necessary to measure a wide range of biological activities for a particular sequence, not just single activities, and for differences (perhaps only subtle) to be appreciated, before conclusions can be drawn. This is related to the recent proposal that HS forms some of the major nodes in networks of interacting proteins that lie at the heart of mammalian signalling systems (3).

The fact that HS structures can be mimicked by nonglycosaminoglycan (GAG) sequences has been appreciated for some time, but the demonstration that they interact in essentially the same way as HS structures and can induce similar structural changes in proteins, as well as comparable protein stabilisation and activities, is more recent $(7,9)$. These findings suggest that non-GAG analogues may also be able to serve as HS mimics, not only for individual activities, but also as agents capable of eliciting several activities, akin to those provided by HS. This may provide an important opportunity for HS derivatives, mimics and analogues to be employed as a means of intervening in biochemical processes for medical purposes. It will be important to eliminate unwanted side effects, the most obvious being anticoagulation through antithrombin and factor $X_{a}$, as well as interactions with the extrinsic and intrinsic clotting pathways, but a range of potential applications are evident (10).

\section{Heparan sulphate is a key player in attachment and invasion of microbial pathogens and is emerging as a target for intervention}

The emerging properties of HS suggest that agents able to mimic particular charge and shape characteristics may be able to elicit similar biological responses and this has indeed been shown to be the case in several experimental systems. Perhaps the most important scope for developing agents capable of interfering with protein-ligand interactions comes in the field of infectious diseases (11). One additional feature of these molecules, which distinguishes them from conventional small molecule drugs, is their sheer size. Not only is there the possibility of mimicking particular interactions with proteins during recognition, binding and invasion, but the size of complex carbohydrates renders them suitable as efficient inhibitors or disruptors of binding events because they have the ability to act at several binding sites simultaneously, or concertedly. The efficiency of such processes is greatly enhanced through the multivalent effect (12) and examples of this occur during the adhesion and invasion of microbial pathogens.

\section{Proteoglycans and microbial pathogenesis}

Proteoglycans (PGs), especially those harbouring the structurally diverse HS chains, bind to and regulate several biological molecules through their GAG chains. These include growth factors, cytokines, chemokines, proteinases, antimicrobial factors, ECM components and many more (13). In many cases, PGs serve as co-receptors that capture ligands and manage the encounter between ligands and their signalling receptors. Another possibility is that PGs regulate protein-protein interactions by affecting the stability, conformation and oligomerisation state of the ligand and receptor. In fact, some pathogens can use their adhesins and virulence factors to increase their pathogenic activities (11). Proteoglycans can also be released from the cell surface or ECM by proteolytic cleavage and become soluble. When this occurs, PGs may behave differently and certain pathogenic bacteria can exploit this to evade the defence system of the host. In fact, some pathogens possess features capable of modulating the expression or function of these proteins to release PGs and render them soluble (14).

\section{Proteoglycans and pathogens}

Many viruses bind to the HS moiety of PGs to improve their attachment and internalization (15). An example is dengue virus, which causes hemorrhagic fever and shock syndrome, and is transmitted to humans by the Aedes aegyptimosquito. Interestingly, dengue virus binds specifically to immobilized heparin and both heparin and HS ligands block infection, suggesting that HSPGs serve as receptors on endothelial cells (16). In fact, when endothelial cells are treated with heparinase III, which cleaves the polysaccharide chains of the HSPGs, infectivity is lost (17). Since the extent of infection of the endothelial cells can have a crucial impact on the severity of the disease, HS may serve as a promising target for controlling the course of the infection.

Other examples are herpes simplex virus (HSV), serotypes 1 and 2. HSV has two glycoproteins on its surface, gB and $\mathrm{gC}$, which bind to cell surface HSPGs and mediate the initial attachment to epithelial, skin, cornea and urogenital cells (18). Most studies suggest that syndecan-1 might be involved in target cell specificity. However, no HSPGs have been identified mediating the HSV binding to target cells, but remain a probable candidate.

It is well known that many bacterial pathogens can bind to GAGs and proteoglycans to promote their binding and even internalization. Pathogenic leptospires, for instance, have been shown to bind to mammalian cell layers in culture and attach to specific ECM components much more efficiently than non-pathogenic, saprophytic, strains (19). 
Breiner et al. (20) tested L. interrogans (serovar Copenhageni) for its binding activity to GAG and proteoglycans and demonstrated that this potent human pathogen can bind strongly to chondroitin sulphate B and C GAGs. In contrast, Borrelia burgdorferi, another pathogenic spirochete, recognizes heparin, HS and chondroitin sulphate $B$, but not chondroitin sulphate $C(21)$. The authors point out that heparin is widely used as a tractable model GAG and is produced by, and released from, mast cells, so it is not normally present in the ECM. On the other hand, HS and chondroitin sulphate are distributed widely and serve as targets for bacterial attachment. For Leptospira, many microbial surface components recognizing adhesive matrix molecules have been identified as possible mediators for ECM molecules (22). Usually, these proteins bind to the repeated elements of the ECM, so it is reasonable that they might also recognize the repeats of GAGs. In fact, for $B$. burgdorferi the fibronectin-binding protein also binds to GAGs (23). For leptospira the proteins involved in the recognition of GAGs still remain to be identified, but adhesins such as LigA, LigB, LipL32, TlyC and other family of proteins (LenA to LenF, Lsa24 and LfhA) are candidates (24-26).

Neisseria gonorrhoeae causes a common, but often asymptomatic sexual transmitted infection (STI). Cell-surface HSPGs serve as co-receptors and direct internalization receptors for this pathogen. Available data suggest that binding of $N$. gonorrhoeae Opa protein to syndecan 1 and 4 is an important step in the pathogenicity of this bacterium. Experiments with cells expressing syndecan 1 and 4 showed that, although the presence of these proteoglycans on the cell surface facilitated bacterial adherence, they did not facilitate the invasion of $N$. gonorrhoeae into the cells when alone. This occurs because intracellular signalling mediated by the cytoplasmic domain of syndecans is essential to promote the gonococcal invasion (27). In vitro assays demonstrated that an alternative invasion route for N. gonorrhoeae is to employ HSPGs as co-receptors to facilitae fibronectin-mediated internalisation in Hep-2 cells (28). The strategy for these bacteria is to use the HSPGs to bind fibronectin, and use fibronectin as a bridge to bind to integrins, which promotes internalisation of the bacteria.

For most parasites, the initial binding steps in adhering to cells are based on HSPG recognition. The efforts made to identify the heparin-binding proteins involved in cell recognition also providing a prospective target for diagnosis or therapy. Trypanosoma cruzi, the causative agent of Chagas' disease, uses a number of different strategies to invade mammalian cells. The invasion process is complex and involves triggering distinct signalling pathways $(29,30)$, since both trypomastigotes and amastigotes are able to invade mammalian cells and sustain the intracellular cycle (31). The molecules needed for amastigote invasion are still not well elucidated, but HSPGs can activate a signalling cascade causing a rearrangement of the cytoskeleton (32), which is associated with the GAG-binding proteins in amastigote forms of T. cruzi (33). Bambino-Medeiros et al. (34) have shown that, when amastigote forms are treated with heparin and heparan sulphate, the invasion of cardiomyocyte cells is prevented by 82 and $65 \%$, respectively.

Most of the examples given illustrate how microorganisms can use GAGs to adhere to or invade host cells, influencing the infection directly. However, another possibility is to use the proteoglycans to evade host defences. It is well known that cationic antimicrobial peptides, such as defensins and cathelicidins are important components of the innate immune response against many pathogens (35) acting by disrupting the lipid membranes of various classes of pathogens including Gram-negative and -positive bacteria, parasites and fungi. It has been hypothesised that they probably exploit negatively charged GAGs to neutralise the effect of cationic antimicrobial peptides, since they are positively charged (35). Staphylococcus aureus, a Gram-positive bacterium responsible for causing infections ranging from dermatitis to meningitis, is able to release syndecan-1 from the cell surface by secreting virulence factors that stimulate the shedding mechanism of the host cell. Metalloproteinases cleave the ectodomain of syndecan- 1 that binds and inhibits those antimicrobial peptides and other host defence factors (36).

Examples of GAG derivatives and GAG mimetics exploiting their capacity to adopt suitable charge and conformational arrangements to interfere with adhesion and act against bacteria (antimicrobial), viruses and parasites have been reported and reviewed (37-40). Important observations are that, not only can sulphated mimics of HS inhibit, or reverse attachment during the infection and disease process but, their efficacy is often strongly dependent on their size (41), supporting the hypothesis that they exhibit a multivalent effect. There are three categories of proteoglycans that can inhibit the pathogen interaction: engineered GAGs (Escherichia coli K5 polysaccharide, modified HS oligosaccharides and periodate-depolymerised heparin); polysulfated compounds (carrageenans, phosphomannopentaose sulphate, rhamnan and curdlan sulphate) and polysulfonated compounds (suramin, sulfonated porphyrin, naphthalene sulfonate polymer). Most of these compounds were tested in viruses, inhibiting their attachment and dissemination to the cells (42), or in parasites, for instance blocking the $P$. falciparum rosette formation in vitro assays (43).

These properties are of obvious relevance to those striving to discover alternative approaches to preventing, or reversing, microbial attachment or invasion. Their ambit ranges from antiviral activity to reversal of rosetting in Plasmodium falciparum malaria. However, it may be even more broadly applicable than previously thought because, for example, recent reports have linked severity in nonfalciparum malaria with an ability to form rosette-like structures in cases of Plasmodium vivax malaria in Brazil (44). 


\section{References}

1. Sampaio LO, Tersariol ILS, Lopes CC, Bouças RI, Nascimento FD, Rocha HAO, et al. Heparins and heparan sulfates. Structure, distribution and protein interactions. In: Verli $\mathrm{H}$ (Editor), Insights into carbohydrate structure and biological function. Kerala: Transworld Research Network; 2006, p 51-61.

2. Ori A, Free P, Courty J, Wilkinson MC, Fernig DG. Identification of heparin-binding sites in proteins by selective labeling. Mol Cell Proteomics 2009; 8: 2256-2265.

3. Ori A, Wilkinson MC, Fernig DG. A systems biology approach for the investigation of the heparin/heparan sulfate interactome. J Biol Chem 2011; 286: 19892-19904.

4. Choay J, Petitou M, Lormeau JC, Sinay P, Casu B, Gatti G. Structure-activity relationship in heparin: a synthetic pentasaccharide with high affinity for antithrombin III and eliciting high anti-factor Xa activity. Biochem Biophys Res Commun 1983; 116: 492-499.

5. Atha DH, Lormeau JC, Petitou M, Rosenberg RD, Choay J. Contribution of monosaccharide residues in heparin binding to antithrombin III. Biochemistry 1985; 24: 6723-6729.

6. Thunberg L, Backstrom G, Lindahl U. Further characterization of the antithrombin-binding sequence in heparin. Carbohydr Res 1982; 100: 393-410.

7. Rudd TR, Uniewicz KA, Ori A, Guimond SE, Skidmore MA, Gaudesi D, et al. Comparable stabilisation, structural changes and activities can be induced in FGF by a variety of HS and non-GAG analogues: implications for sequence-activity relationships. Org Biomol Chem 2010; 8: 5390-5397.

8. Yates EA, Guimond SE, Turnbull JE. Highly diverse heparan sulfate analogue libraries: providing access to expanded areas of sequence space for bioactivity screening. J Med Chem 2004; 47: 277-280.

9. Uniewicz KA, Ori A, Xu R, Ahmed Y, Wilkinson MC, Fernig $D G$, et al. Differential scanning fluorimetry measurement of protein stability changes upon binding to glycosaminoglycans: a screening test for binding specificity. Anal Chem 2010; 82: 3796-3802.

10. Solari V, Jesudason EC, Turnbull JE, Yates EA. Determining the anti-coagulant-independent anti-cancer effects of heparin. Br J Cancer 2010; 103: 593-594.

11. Bartlett AH, Park PW. Proteoglycans in host-pathogen interactions: molecular mechanisms and therapeutic implications. Expert Rev Mol Med 2010; 12: e5.

12. Kitov PI, Bundle DR. On the nature of the multivalency effect: a thermodynamic model. J Am Chem Soc 2003; 125: 16271-16284.

13. Tumova S, Woods A, Couchman JR. Heparan sulfate chains from glypican and syndecans bind the Hep II domain of fibronectin similarly despite minor structural differences. $J$ Biol Chem 2000; 275: 9410-9417.

14. Filmus J, Selleck SB. Glypicans: proteoglycans with a surprise. J Clin Invest 2001; 108: 497-501.

15. Spillmann D. Heparan sulfate: anchor for viral intruders? Biochimie 2001; 83: 811-817.

16. Germi R, Crance JM, Garin D, Guimet J, Lortat-Jacob H, Ruigrok RW, et al. Heparan sulfate-mediated binding of infectious dengue virus type 2 and yellow fever virus. Virology 2002; 292: 162-168.

17. Dalrymple N, Mackow ER. Productive dengue virus infection of human endothelial cells is directed by heparan sulfatecontaining proteoglycan receptors. J Virol 2011; 85: 94789485.

18. O'Donnell CD, Tiwari $\mathrm{V}$, Oh MJ, Shukla D. A role for heparan sulfate 3-O-sulfotransferase isoform 2 in herpes simplex virus type 1 entry and spread. Virology 2006; 346: 452-459.

19. Barocchi MA, Ko Al, Reis MG, McDonald KL, Riley LW. Rapid translocation of polarized MDCK cell monolayers by Leptospira interrogans, an invasive but nonintracellular pathogen. Infect Immun 2002; 70: 6926-6932.

20. Breiner DD, Fahey M, Salvador R, Novakova J, Coburn J. Leptospira interrogans binds to human cell surface receptors including proteoglycans. Infect Immun 2009; 77: 55285536.

21. Leong JM, Morrissey PE, Ortega-Barria E, Pereira ME, Coburn J. Hemagglutination and proteoglycan binding by the Lyme disease spirochete, Borrelia burgdorferi. Infect Immun 1995; 63: 874-883.

22. Patti JM, Hook M. Microbial adhesins recognizing extracellular matrix macromolecules. Curr Opin Cell Biol 1994; 6: 752-758.

23. Fischer JR, LeBlanc KT, Leong JM. Fibronectin binding protein BBK32 of the Lyme disease spirochete promotes bacterial attachment to glycosaminoglycans. Infect Immun 2006; 74: 435-441.

24. Ballard SA, Williamson M, Adler B, Vinh T, Faine S. Interactions of virulent and avirulent leptospires with primary cultures of renal epithelial cells. J Med Microbiol 1986; 21: 59-67.

25. Choy HA, Kelley MM, Chen TL, Moller AK, Matsunaga J, Haake DA. Physiological osmotic induction of Leptospira interrogans adhesion: LigA and LigB bind extracellular matrix proteins and fibrinogen. Infect Immun 2007; 75: 24412450.

26. Carvalho E, Barbosa AS, Gomez RM, Cianciarullo AM, Hauk $P$, Abreu PA, et al. Leptospiral TlyC is an extracellular matrixbinding protein and does not present hemolysin activity. FEBS Lett 2009; 583: 1381-1385.

27. Freissler E, Meyer auf der HA, David G, Meyer TF, Dehio C. Syndecan-1 and syndecan-4 can mediate the invasion of OpaHSPG-expressing Neisseria gonorrhoeae into epithelial cells. Cell Microbiol 2000; 2: 69-82.

28. van Putten JP, Duensing TD, Cole RL. Entry of OpaA+ gonococci into HEp-2 cells requires concerted action of glycosaminoglycans, fibronectin and integrin receptors. $\mathrm{Mol}$ Microbiol 1998; 29: 369-379.

29. Fernandes AB, Neira I, Ferreira AT, Mortara RA. Cell invasion by Trypanosoma cruzi amastigotes of distinct infectivities: studies on signaling pathways. Parasitol Res 2006; 100 : 59-68.

30. Yoshida N, Cortez M. Trypanosoma cruzi: parasite and host cell signaling during the invasion process. Subcell Biochem 2008; 47: 82-91.

31. Ley V, Andrews NW, Robbins ES, Nussenzweig V. Amastigotes of Trypanosoma cruzi sustain an infective cycle in mammalian cells. J Exp Med 1988; 168: 649-659.

32. Dreyfuss JL, Regatieri CV, Jarrouge TR, Cavalheiro RP, Sampaio LO, Nader HB. Heparan sulfate proteoglycans: structure, protein interactions and cell signaling. An Acad 
Bras Cienc 2009; 81: 409-429.

33. Oliveira FO Jr, Alves CR, Calvet CM, Toma L, Boucas RI, Nader HB, et al. Trypanosoma cruzi heparin-binding proteins and the nature of the host cell heparan sulfate-binding domain. Microb Pathog 2008; 44: 329-338.

34. Bambino-Medieros R, Oliveira FO, Calvet CM, Vicente D, Toma L, Krieger MA, et al. Involvement of host cell heparan sulfate proteoglycan in Trypanosoma cruzi amastigote attachment and invasion. Parasitol 2011; 138: 593-601.

35. Lai $Y$, Gallo RL. AMPed up immunity: how antimicrobial peptides have multiple roles in immune defense. Trends Immunol 2009; 30: 131-141.

36. Park PW, Foster TJ, Nishi E, Duncan SJ, Klagsbrun M, Chen Y. Activation of syndecan-1 ectodomain shedding by Staphylococcus aureus alpha-toxin and beta-toxin. J Biol Chem 2004; 279: 251-258.

37. Romanos MT, Andrada-Serpa MJ, Mourão PA, YoneshigueValentin Y, Costa SS, Pereira MS, et al. A sulphated fucan from the Laminaria abyssalis inhibits the human T cell lymphotropic virus type 1-induced syncytium formation in HeLa cells. Antivir Chem Chemother 2002; 13: 219-221.

38. Adams Y, Smith SL, Schwartz-Albiez R, Andrews KT. Carrageenans inhibit the in vitro growth of Plasmodium falciparum and cytoadhesion to CD36. Parasitol Res 2005; 97: 290-294.
39. Buck CB, Thompson CD, Roberts JN, Muller M, Lowy DR, Schiller JT. Carrageenan is a potent inhibitor of papillomavirus infection. PLoS Pathog 2006; 2: e69.

40. Talarico LB, Damonte EB. Interference in dengue virus adsorption and uncoating by carrageenans. Virology 2007; 363: 473-485.

41. Skidmore MA, Dumax-Vorzet AF, Guimond SE, Rudd TR, Edwards EA, Turnbull JE, et al. Disruption of rosetting in Plasmodium falciparum malaria with chemically modified heparin and low molecular weight derivatives possessing reduced anticoagulant and other serine protease inhibition activities. J Med Chem 2008; 51: 1453-1458.

42. Rusnati M, Urbinati C. Polysulfated/sulfonated compounds for the development of drugs at the crossroad of viral infection and oncogenesis. Curr Pharm Des 2009; 15: 29462957.

43. Kyriacou HM, Steen KE, Raza A, Arman M, Warimwe G, Bull $\mathrm{PC}$, et al. In vitro inhibition of Plasmodium falciparum rosette formation by Curdlan sulfate. Antimicrob Agents Chemother 2007; 51: 1321-1326.

44. Carvalho BO, Lopes SC, Nogueira PA, Orlandi PP, Bargieri DY, Blanco YC, et al. On the cytoadhesion of Plasmodium vivax-infected erythrocytes. J Infect Dis 2010; 202: 638647. 